\title{
Molecular processes that handle - and mishandle — dietary lipids
}

\author{
Kevin Jon Williams \\ Division of Endocrinology, Diabetes, and Metabolic Diseases, Department of Medicine, \\ Jefferson Medical College, Thomas Jefferson University, Philadelphia, Pennsylvania, USA.
}

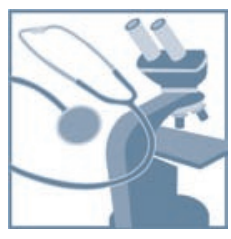

\begin{abstract}
Overconsumption of lipid-rich diets, in conjunction with physical inactivity, disables and kills staggering numbers of people worldwide. Recent advances in our molecular understanding of cholesterol and triglyceride transport from the small intestine to the rest of the body provide a detailed picture of the fed/fasted and active/sedentary states. Key surprises include the unexpected nature of many pivotal molecular mediators, as well as their dysregulation - but possible reversibility - in obesity, diabetes, inactivity, and related conditions. These mechanistic insights provide new opportunities to correct dyslipoproteinemia, accelerated atherosclerosis, insulin resistance, and other deadly sequelae of overnutrition and underexertion.
\end{abstract}

\section{Introduction}

Why has intestinally absorbed lipid remained the focus of clinical and basic efforts spanning at least a century (1)? Because absorption of dietary essential fatty acids and lipid micronutrients by the small intestine sustains human life, but at the same time, the capacity of this organ to absorb our flagrantly lipid-rich modern diet maims and kills people. It does so in at least 3 major ways. First, intestinally derived lipoproteins, chiefly chylomicrons (CMs), transport calorie-dense triglycerides to adipose depots (2, 3 ), accelerating the development of obesity (2) and its many harmful sequelae. Second, while delivering triglycerides to peripheral tissues, CMs become processed into so-called CM "remnants" that carry significant amounts of cholesterol. These remnant particles, particularly the smaller ones (4), enter the arterial wall and are retained there (5), initiating and promoting the development of atherosclerotic plaques (6). Third, CMs and their remnants interfere with the catabolism of harmful hepatic lipoproteins (7, $8)$ that are also retained within the vessel wall, promoting atherogenesis $(6,9-12)$. Postprandial dyslipidemia, which refers to the abnormal persistence of intestinal and hepatic lipoproteins in plasma after each meal, makes a substantial - and potentially avoidable - contribution to the population-wide burden of atherosclerotic cardiovascular disease, particularly in diabetes and related conditions $(4,13)$. Atherosclerosis kills roughly half of individuals living in Westernized countries and 30\% of people worldwide, making it easily the most deadly human disease (14). The overfed intestine drives much of the problem.

This review follows the path of dietary and biliary lipid once it enters the lumen of the small intestine (Figure 1). The focus is lipid macronutrients, chiefly triglycerides and cholesterol, because they are causally linked to death and disability from obesity and cardiovascular disease. Nonlipid calories are not covered, although their

Nonstandard abbreviations used: ABC, ATP-binding cassette transporter; ACAT2, acyl-CoA:cholesterol acyltransferase-2; CM, chylomicron; DGAT, acyl-CoA:diacylglycerol acyltransferase; GPI, glycosylphosphatidylinositol; GPIHBP1, GPI-anchored HDLbinding protein 1; HSPG, heparan sulfate proteoglycan; LpL, lipoprotein lipase; MTP, microsomal triglyceride transfer protein; NEFA, nonesterified fatty acid; NPC1L1,

Niemann-Pick C1-like 1 protein; UC, unesterified cholesterol.

Conflict of interest: The author has declared that no conflict of interest exists.

Citation for this article: J. Clin. Invest. 118:3247-3259 (2008). doi:10.1172/JCI35206. effects on lipid and lipoprotein biology can be found elsewhere (8, $15)$. Strikingly, the molecular responses to obesity, insulin resistance, physical inactivity, and related conditions paradoxically enhance intestinal CM production, direct CM triglycerides away from combustion in striated muscle and toward storage in adipose tissue, and worsen atherogenic postprandial dyslipidemia (Table 1 ). Nevertheless, most, if not all, of these points of molecular dysregulation may be reversible.

Facilitated absorption of cholesterol: from drug to target The major recent breakthrough in understanding cholesterol absorption by the small intestine arose from the discovery of ezetimibe, a medicine that blocks this pathway (16). Notably, the medicine was found via low-throughput screens in whole animals; moreover, it was FDA approved and on the market for years before its molecular target was identified. Ezetimibe provides a compelling counterexample against the prevailing postgenome paradigm for drug discovery, dubbed conveyor belts, in which high-throughput screens of compounds against known protein targets lead to FDA-approved medicines.

Despite some initial controversy $(17,18)$, it is clear that the target of ezetimibe is the Niemann-Pick C1-like 1 protein (NPC1L1; refs. $17,19)$. The NPC1L1 protein is highly expressed in small intestine (19) and in human liver (20), precisely where the drug acts. Ezetimibe glucuronide binds to a single, saturable site on wild-type enterocyte brush border membranes and to cells expressing recom-

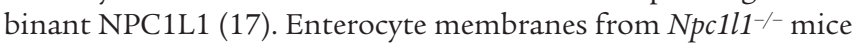
show no ezetimibe binding (17), and Npc111-deficient mice exhibit an approximately $70 \%$ reduction in cholesterol absorption that cannot be lowered further by administration of ezetimibe (19). In humans, NPC1L1 gene variants correlate with sterol absorption (21), baseline plasma LDL concentrations (21), and responsiveness of LDL levels to ezetimibe administration (18). Moreover, most human NPC1L1 variants associated with inefficient intestinal cholesterol absorption exhibit substantial impairments in protein expression, consistent with the phenotype (21).

Physiologic regulation of NPC1L1 alters cholesterol absorption in response to availability, apparently through a sterol-regulatory element in the promoter and a sterol-sensing domain in the protein. Cholesterol-rich diets suppress Npc1l1 expres- 


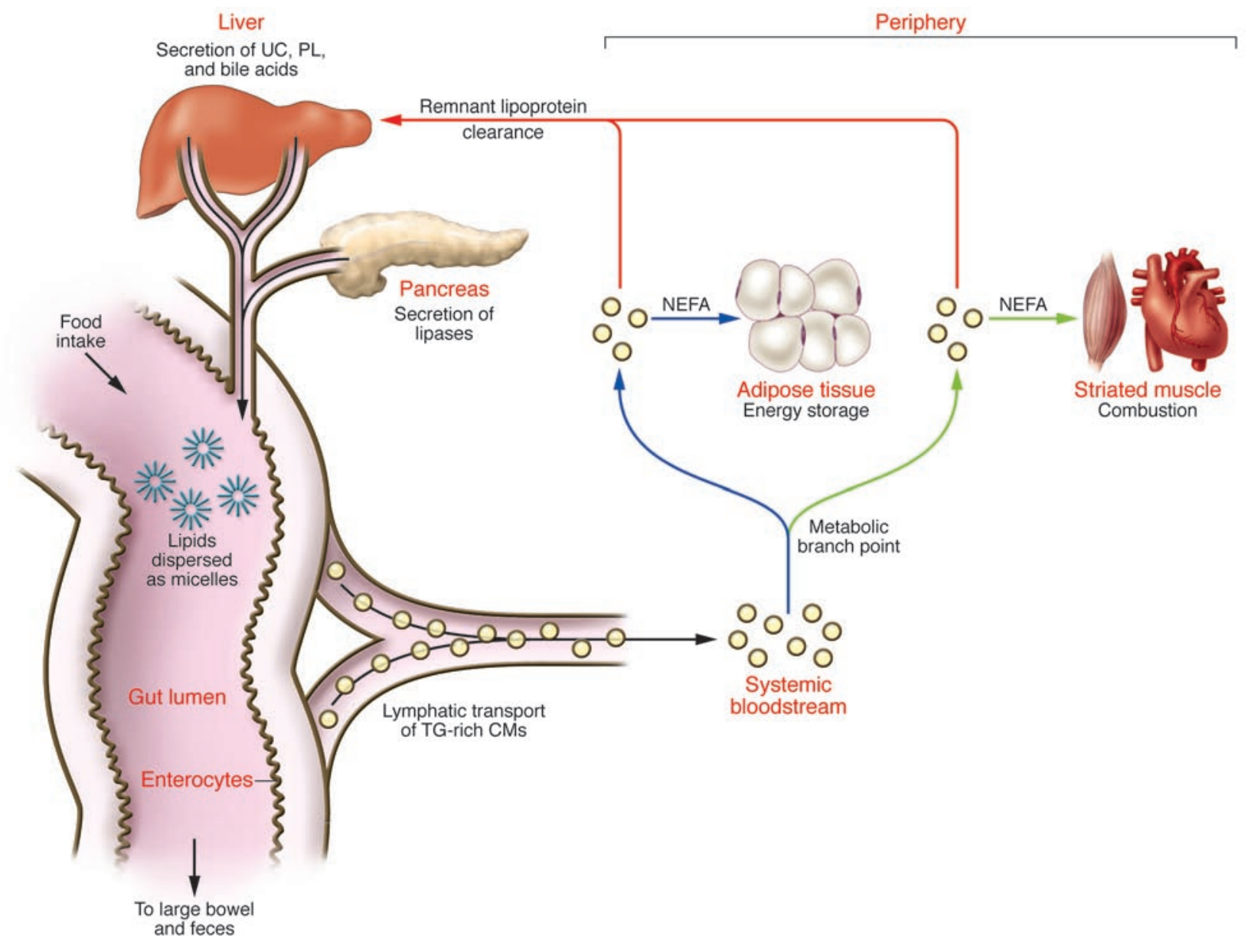

Figure 1

Overview of lipid transport from gut lumen to periphery to liver. Detergents in hepatic bile, chiefly phospholipids (PL) and bile acids, emulsify lipids from food to form microscopic micelles within the gut lumen. Hepatic bile also contributes significant amounts of UC to these micelles. The pancreas secretes lipases that digest dietary lipids into chemical forms that can be absorbed by the gut epithelium, e.g., NEFAs, monoacylglycerides, and UC. Absorption of fatty acids and monoacylglycerides approaches $100 \%$ efficiency and occurs via passive diffusion and carrier-mediated processes (64). Cholesterol absorption averages about 50\% efficiency with considerable interindividual variation. Enterocytes reesterify these lipids intracellularly and then package them into large particles, CMs, that are rich in triglycerides (TG) but also contain substantial amounts of $\mathrm{UC}$ and cholesteryl esters. These particles are secreted into thoracic lymph, which drains directly into the systemic bloodstream, bypassing the liver. The mixed lipid composition of CMs leads to a 2-step clearance process. First, when these particles reach the capillary beds of peripheral tissues, a key metabolic branch point occurs between energy storage in adipose tissue (blue arrows) and lipid combustion in striated muscle (green arrows). In a regulated fashion, CMs dock on the microvascular endothelium of these tissues, where LpL hydrolyzes CM triglycerides into NEFAs to deliver lipid calories for local use. In the second step of clearance, residual triglyceride-depleted, cholesteryl ester-rich particles, now called CM remnant lipoproteins, are released back into plasma. Under normal circumstances, the liver rapidly and safely removes them from the circulation (red arrows).

sion in murine intestine, and cholesterol depletion enhances its expression in porcine intestine and liver (18). Posttranslationally, NPC1L1 moves from internal membranes into the plasma membrane during cholesterol deprivation, facilitating absorption (22). These studies of translocation might reconcile reports that Npc111 is (19) or is not (23) found in the enterocyte brush border. Consistent with translocation, the predominant effect of NPC1L1 appears to be the facilitated trafficking of unesterified cholesterol (UC) from the plasma membrane into the ER, where the lipid becomes esterified and packaged into nascent lipoproteins (Figure 2 and ref. 24). In contrast, the effect of NPC1L1 on cholesterol movement into the plasma membrane from exogenous sources is modest (24).
The NPC1L1 protein exerts major effects on the transport of dietary and nondietary cholesterol within the gastrointestinal system. Even in individuals consuming a lipid-rich modern diet, more than two-thirds of the cholesterol that enters the gut lumen comes from bile (Figure 1 and ref. 21). Thus, by blocking NPC1L1 in the intestine, ezetimibe not only impairs absorption of dietary cholesterol, but also depletes cholesterol from the enterohepatic circulation. Liver cholesterol content drops, an effect augmented clinically by coadministration of statins. Consequences include a slight impairment in hepatic VLDL secretion and a substantial induction of sterol-responsive genes in the liver, particularly that encoding the LDL receptor (18). These effects lower plasma LDL levels by $15 \%-20 \%$. 
Table 1

Dysregulated mediators of lipid and lipoprotein trafficking in diabetes, obesity, and related conditions

$\begin{array}{lc}\begin{array}{l}\text { Molecule/process } \\ \text { NPC1L1, ABCG5/G8 }\end{array} & \begin{array}{c}\text { Dysregulation } \\ \text { In diabetes, increased intestinal and hepatic } \\ \text { expression of Npc1l1 (28) and decreased } \\ \text { intestinal expression of ABCG5/G8 (28-30) } \\ \text { Intestinal overexpression in intestinal } \\ \text { insulin resistance (15) and diabetes (28, 30) } \\ \text { Increased synthesis of fatty acids, triglycerides, } \\ \text { and cholesteryl esters by enterocytes } \\ \text { in intestinal insulin resistance (15) }\end{array} \\ \text { Intestinal lipid synthesis } & \begin{array}{c}\text { Increased activity in adipose tissue, but decreased } \\ \text { activity in striated muscle, in the fed } \\ \text { and sedentary states (3, 78, 79) } \\ \text { Suppressed expression in adipose } \\ \text { tissue in the fed state (84) }\end{array} \\ \text { Mitochondrial } \beta \text {-oxidation } & \text { Reduced capacity in sedentary skeletal muscle (86) }\end{array}$

Gpihbp1

Suppression of its mRNA in muscle in the fed state (90)

CD36 fatty acid translocase Ndst1

\author{
Likely consequences \\ Increased intestinal cholesterol absorption, decreased \\ hepatic biliary cholesterol excretion, and increased \\ cholesterol content of secreted CMs and VLDL \\ Substantially increased intestinal secretion of $\mathrm{apoB}_{48}$, \\ hence an increased number of secreted particles \\ Increased CM secretion in insulin \\ resistance and overt diabetes (67) \\ Diversion of CM and VLDL calories into \\ adipose depots for storage and away \\ from combustion in muscle \\ Diversion of CM and VLDL calories into adipose depots \\ owing to a local increase in enzymatically active $L p L$ dimers \\ Accumulation of triglycerides and fatty acid \\ metabolites, leading to insulin resistance in muscle \\ Diversion of CM and VLDL calories away \\ from combustion in muscle
}

Enhanced adipose uptake of calories in the form of NEFAs

Defective hepatic assembly of HSPGs, hence abnormal persistence of atherogenic postprandial remnant lipoproteins in plasma

Ndst1, heparan sulfate $N$-deacetylase/ $N$-sulfotransferase-1.

In transgenic mice, ezetimibe also blocks NPC1L1 within the liver, thereby allowing endogenous cholesterol to exit into bile rather than being taken back up into hepatocytes for secretion into plasma (25). Because human liver abundantly expresses NPC1L1 (20), clinical administration of ezetimibe might also increase cholesterol elimination into bile (25). Thus, blockage of NPC1L1 augments the ability of the gastrointestinal system to dispose of dietary and nondietary cholesterol. For example, reverse transport of cholesterol, from lipid-loaded peripheral macrophages to HDL to the liver, then into bile and out into the feces, becomes more efficient in ezetimibe-treated mice, owing to inhibition of intestinal reabsorption of HDL-derived biliary cholesterol, but without any apparent effects on macrophages or on HDL particles $(26,27)$.

Regarding dyslipidemias, type 1 diabetic rats show increased Npc111 expression in both intestine and liver, and the levels correlate, respectively, with CM and VLDL cholesterol content (Table 1 and ref. 28). This finding may contribute to cholesterol hyperabsorption in human type 1 diabetes (29). Type 2 diabetes also increases intestinal NPC1L1 mRNA in humans (30), and among type 2 diabetic patients, those with coronary artery disease absorb cholesterol more efficiently than those without (31). Compared with nondiabetic controls, however, type 2 diabetic subjects exhibit increased intestinal cholesterol synthesis, not absorption (29). In the other direction, genetic deficiency of Npc1l1 in the apoE knockout mouse, an animal model that accumulates remnantlike lipoproteins in its plasma, lowers the cholesterol content of atherogenic lipoproteins by $80 \%-90 \%$ and blocks the development of vascular disease (32). Regarding human atherosclerosis, long-term studies to examine the effectiveness of ezetimibe on cardiovascular outcomes must still be completed, even though nearly every strategy to lower plasma LDL levels has resulted in essentially the same degree of long-term cardiovascular protection (11). A 2-year study in familial hypercholesterolemic subjects failed to find a significant effect of this medicine on carotid intimal-medial thickness (33).

Surprisingly, the expression of NPC1L1 by enterocytes also facilitates uptake of toxic, noncholesterol sterols from plants, shellfish, and other dietary sources $(17,18)$. The ability of the normal intestine to selectively prevent these molecules from moving into the rest of the body was recognized decades ago with the discovery of $\beta$-sitosterolemia, a rare recessive disease in which plant sterols accumulate systemically. The responsible mutations reside in either of 2 ATP-binding cassette transporters (ABCs), ABCG5 and ABCG8 (34). These proteins are actually half-transporters that form heterodimers (35) that pump nearly all noncholesterol sterols out of the enterocyte, back into the gut lumen for excretion in feces (Figure 2 and ref. 34). The ABCG5/G8 transporter also pumps some cholesterol out of enterocytes (34), thereby explaining cholesterol hyperabsorption in $\beta$-sitosterolemia. Suppression of ABCG5/G8 in type 1 diabetic intestine may also contribute to cholesterol hyperabsorption (Table 1 and refs. 28, 29).

Dimers of Abcg5/g8 are also expressed in liver, where they cooperate with Abcb4 (also known as Mdr2) to pump UC and phospholipid into bile (34), while hepatic NPC1L1 transports UC from bile back into the hepatocyte (25). The combined effects of these 4 proteins regulate biliary cholesterol content before its release into the intestinal lumen. Both Abcg5 and Abcg8 are liver $\mathrm{X}$ receptor (Lxr) targets and are induced by high-cholesterol diets. Lxr agonists enhance intestinal cholesterol excretion through these transporters (36); but, as side effects, these agonists also induce lipid biosynthesis and steatosis in the liver as well as apoptosis of pancreatic $\beta$ cells. 


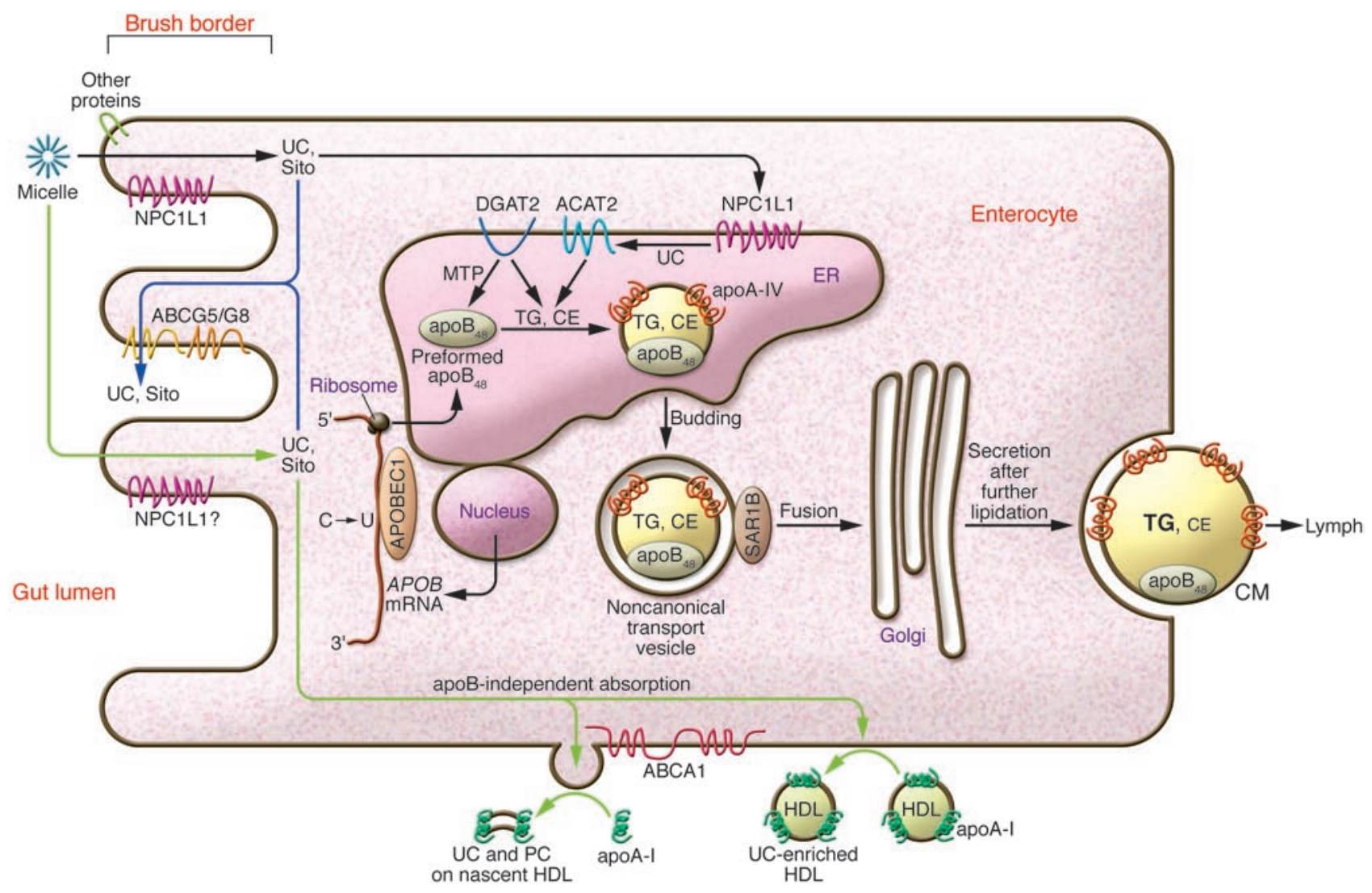

Figure 2

Processing of dietary lipid by the enterocyte: absorption, selection, packaging, and secretion. In the apoB-dependent pathway (black arrows), $\mathrm{apoB}_{48}$ becomes assembled in the ER with initially small amounts of triglycerides that are synthesized mainly by DGAT2 and transferred via MTP. UC imported from the gut lumen into the enterocyte via NPC1L1 and possibly other proteins becomes esterified by ACAT2 and also packaged into the newly forming $\mathrm{apoB}_{48}$ lipoprotein. Continued lipidation in the ER inflates the particle, requiring stabilization of its surface by phospholipids and apoA-IV. The nascent particles are then transferred to the Golgi apparatus in noncanonical transport vesicles, further lipidated, and finally secreted as CMs into lymph. Some of the absorbed cholesterol and essentially all of the dietary noncholesterol sterols are pumped out of the enterocyte, back into the intestinal lumen for elimination from the body (blue arrows). In the apoB-independent pathway (green arrows), UC is absorbed into the enterocyte, and then ABCA1 facilitates its efflux from the plasma membrane onto extracellular apoA-I molecules. Movement onto preexisting extracellular HDL also occurs. The apoA-I originates both from local intestinal synthesis and from plasma. Absorption of NEFAs, monoglycerides, and lipid micronutrients across the brush border and esterification by acyl-CoA:monoacylglycerol acyltransferase enzymes are not shown here. CE, cholesteryl ester; PC, phosphatidylcholine; Sito, sitosterol and other noncholesterol sterols.

\section{Intestinal packaging of absorbed lipids into apoB $_{48}$-containing lipoproteins}

Similarities to hepatic production of apoB ${ }_{100}$-containing lipoproteins A key structural component of CMs is the huge, hydrophobic, nonexchangeable protein apoB, which exists in 2 forms in the body. Human liver makes full-length apoB ${ }_{100}$, the major protein of hepatically derived atherogenic lipoproteins (e.g., VLDL and $\mathrm{LDL}$ ). The $\mathrm{C}$ terminus of $\mathrm{apoB}_{100}$ contains the domain recognized by the LDL receptor, thereby mediating high-affinity clearance of these particles from plasma. In contrast, essentially all of the apoB from human intestine appears as a truncated form, $\mathrm{apoB}_{48}$, that is missing the C-terminal 52\% (37), and so CMs and CM remnants rely on exchangeable proteins, particularly lipoprotein lipase (LpL; refs. 38-41), hepatic lipase $(42,43)$, and apoE (44-46), to serve as ligands for cell surface receptors. Polymorphisms of each of these exchangeable proteins affect fasting lipoprotein concentrations (47) and the postprandial response (43). Intestinal apoB 48 comes from the same gene as $\mathrm{apoB}_{100}$ through the action of the $A P O B$ mRNA editing complex-1 (APOBEC1), which deaminates a single cytidine into a uridine, thereby creating a nongenomic inframe stop codon (UAA) roughly in the middle of the RNA coding region (Figure 2 and ref. 48).

Far more details are known about the cellular processes in the liver that allow this huge, hydrophobic protein to become cotranslationally and posttranslationally assembled with lipids (49) and what regulates its subsequent secretion (50). Both intestine and liver depend on acyl-CoA:diacylglycerol acyltransferases (DGATs) and acyl-CoA:cholesterol acyltransferase-2 (ACAT2) for intracellular synthesis of triglycerides and cholesteryl esters that will be assembled into the hydrophobic cores of nascent apoB-containing lipoproteins (Figure 2). Both organs require the microsomal triglyceride transfer protein (MTP) to shuttle small amounts of triglyceride onto each newly forming apoB molecule as it translocates into the ER lumen, thereby allowing proper folding of the protein. A second lipidation step later in the secretory 
pathway occurs in enterocytes $(51)$ and in hepatocytes $(52,53)$ to generate large CMs and VLDL, respectively. In hepatocytes, extensive lipidation of nascent apoB lipoproteins requires a motif that is present in both $\mathrm{apoB}_{48}$ and $\mathrm{apoB}_{100}$ (53), but a role for this motif has not yet been reported in enterocytes. Fully assembled apoB lipoproteins are released by exocytosis of secretory vesicles from the basolateral membrane of enterocytes (Figure 2).

Genetic deficiency or pharmacologic inhibition of MTP allows continued synthesis of apoB $(49,54)$, but the misfolded protein becomes destroyed by ER-associated degradation (ERAD), a process mediated in cultured hepatocytes by the proteasome (49). The same mechanism is presumably at work in MTP-deficient enterocytes. Genetic lack of MTP causes abetalipoproteinemia, a heritable condition characterized by the complete absence of $\mathrm{apoB}_{48}$ and $\mathrm{apoB}_{100}$ lipoproteins from plasma, as well as the development of intestinal and hepatic steatosis, malabsorption, and deficiencies of fat-soluble vitamins (55). Unfortunately, the side effects of fatty intestine and liver hamper the use of pharmacologic inhibitors of MTP to lower plasma apoB lipoprotein levels in humans (56). However, in unmanipulated intestinal cells (54) and in normal primary hepatocytes (57), ERAD appears to have little or no role in the physiologic regulation of apoB secretion. Fatty meals induce Mtp expression in normal enterocytes, presumably to facilitate lipid absorption and packaging (58).

In collaboration with the Fisher laboratory, my colleagues and I identified 2 new processes that regulate apoB secretion from cultured hepatocytes and from liver in vivo: post-ER presecretory proteolysis $(50,57,59)$ and reuptake $(39,50,60)$, both of which could be active in intestine. Post-ER presecretory proteolysis is of particular interest, given our finding that polyunsaturated $\Omega-3$ fatty acids from fish oils preferentially inhibit hepatic secretion of large apoB lipoproteins via this mechanism (50). Chronic administration of these same fatty acids blunts postprandial hypertriglyceridemia in humans via decreased CM production (61).

\section{Unique features of intestinal production of apoB $_{48}$-containing lipoproteins}

The intestine has a unique need to rapidly assemble and secrete gigantic CM particles to carry large amounts of newly absorbed triglycerides. Several adaptations have been identified, the first of which is $\mathrm{apoB}_{48}$ itself. Comparisons of wild-type and Apobec1-/mice, which cannot edit intestinal Apob mRNA, revealed that the short form of apoB assembles more efficiently into CMs than does $a_{p o B} B_{100}$, particularly after a lipid-rich meal (48). To quickly export triglycerides after dietary absorption, the intestinal mucosa appears to keep a preformed supply of $\mathrm{apoB}_{48}$ in reserve that becomes lipidated into large CMs as needed (62). Recruitment of apoB ${ }_{48}$ from this preformed pool may involve MTP. Accordingly, a substantial increase in $\mathrm{apoB}_{48}$ secretion occurs in conditions with abnormally high intestinal MTP expression. Examples include the $-493 \mathrm{~T}$ human MTP promoter polymorphism (43), intestinal insulin resistance (15), and overt diabetes $(28,30)$.

Fat feeding also provokes marked intestinal expression of an amphipathic protein, apoA-IV, that serves as a surface component for the rapidly inflating $\mathrm{apoB}_{48}$ particles within the enterocyte (Figure 2 and refs. 63, 64). Once CMs reach the bloodstream, apoA-IV becomes displaced by apoCs and apoE as a prelude to lipolysis and clearance. A common apoA-IV variant that exhibits higher lipid affinity, Q360H, has been associated with increased postprandial hypertriglyceridemia, presumably by impeding this transfer of apoCs and apoE onto CMs (63).
CMs vastly exceed the size of canonical ER-to-Golgi transport vesicles, which are only 60-70 $\mathrm{nm}$ in diameter (65). In hepatocytes, 2 adaptations have been reported: slightly larger transport vesicles of about $100 \mathrm{~nm}$ and the release of consistently small, dense $a_{100} B_{100}$ particles from the ER regardless of lipid availability (52). Several lines of evidence, however, indicate that intestinal adaptations possess distinct features. First, the enterocyte performs considerable lipidation of $\mathrm{apoB}_{48}$ particles in the ER, and as a consequence, the ER-to-Golgi transport vesicles have been reported to average about $250 \mathrm{~nm}$ in diameter (64), over 50 times the volume of canonical transport vesicles (Figure 2). Second, to create and then target these unusual transport vesicles, the enterocyte reportedly uses a unique set of proteins. Instead of depending on coat proteins (COPII) for ER budding, the enterocyte apparently relies on intracellular fatty acid-binding protein-1 (Fabp1), and perhaps Fabp2, to create $\mathrm{apoB}_{48}$ transport vesicles (64). Of note, the A54T polymorphism of FABP2 associates with increased postprandial lipemia (43). Third, a rare inherited syndrome, CM retention disease, preferentially affects the intracellular transport and secretion of $\mathrm{apoB}_{48}$ lipoproteins from enterocytes while still permitting secretion of $a_{p o B} B_{100}$ particles from liver. Patients exhibit malabsorption, fat-filled enterocytes, no plasma $\mathrm{apoB}_{48}$, no postprandial $\mathrm{CMs}$, present but decreased plasma levels of $\mathrm{apoB}_{100}$ lipoproteins, and low HDL levels. Jones et al. (65) and others (66) linked this syndrome with several mutations in the SAR1B protein, a GTPase involved in the budding of canonical COPII-coated vesicles from the ER. In hepatocytes, Sar1 proteins are required for these apoBcontaining vesicles to bud off the ER (52). In an enterocyte system, however, blockage of Sar1 still allowed the generation of $\mathrm{apoB}_{48}$ transport vesicles, presumably in the context of continued activity of intracellular Fabp1 and Fabp2, but these abnormal vesicles failed to fuse with the Golgi complex (64). Based on these differences, it is possible that SAR1B mutations in CM retention disease allow budding, transport, and Golgi fusion of apoB-containing vesicles in hepatocytes but interfere with these processes, particularly Golgi fusion, in enterocytes. This overall cellular and molecular understanding has led to several strategies to inhibit apoBdependent absorption of lipids from the gut lumen, some under exploration in vivo and others already in clinical use (Table 2).

Because human liver makes no $\mathrm{apoB}_{48}$ and human intestine synthesizes no detectable $a p B_{100}(37)$, the type of apoB identifies the origin of an atherogenic human lipoprotein. Each of these lipoproteins contains exactly 1 molecule of apoB, and so the mass of $\mathrm{apoB}_{48}$ or $\mathrm{apoB}_{100}$ is used to estimate particle number. After a fatty meal, roughly $80 \%$ of the rise in plasma triglyceride concentration in humans is carried by apoB 48 lipoproteins, chiefly large $\mathrm{CMs}$, but about $80 \%$ of the increase in particle count comes from apoB $\mathrm{B}_{100}$ lipoproteins (8). The comparatively low number of $\mathrm{apoB}_{48}$ particles has led some authors to propose that the effects of intestinal $\mathrm{apoB}_{48}$ lipoproteins on vascular disease are largely indirect, brought about by their ability to increase the concentration and atherogenicity of $\mathrm{apoB}_{100}$ lipoproteins and to lower plasma HDL levels $(7,8)$. In intestinal insulin resistance and overt diabetes, the increase in $\mathrm{apoB}_{48}$ secretion noted above implies a higher particle number. Insulin resistance also enhances endogenous intestinal synthesis of fatty acids, triglycerides, and cholesteryl esters that contribute to CM overproduction $(15,67)$ and downstream atherogenic effects (Table 1 and ref. 7).

The intestine also mediates several pathways for lipid absorption that do not depend on apoB. For example, some cholesterol absorption occurs via HDL and its major protein, apoA-I (Figure 2 


\section{Table 2}

Pharmacologic strategies to inhibit apoB-dependent absorption of lipids from the gut lumen

\begin{tabular}{|c|c|}
\hline Target and strategy & Rationale \\
\hline Additional NPC1L1 inhibitors & $\begin{array}{l}\text { Reduced intestinal absorption of biliary and dietary cholesterol, particularly in the } \\
\text { subset of poor responders to the currently available NPC1L1 inhibitor ezetimibe }\end{array}$ \\
\hline Pancreatic lipase inhibitors & $\begin{array}{l}\text { Impaired digestion and absorption of dietary triglycerides; better adherence to low-fat diets owing } \\
\text { to abdominal discomfort after eating oils that cannot be absorbed; agents are in clinical use }\end{array}$ \\
\hline MGAT2 inhibition & Impaired reassembly of triglycerides within the enterocytes, which could impede CM assembly \\
\hline DGAT1 inhibition & $\begin{array}{l}\text { Dgat } 1^{-1-} \text { mice exhibit reduced postprandial chylomicronemia, increased energy expenditure, and resistance } \\
\text { to obesity, all of which would be desirable characteristics; conversely, Dgat2-- mice die as neonates }\end{array}$ \\
\hline ACAT2 inhibition & Reduced assembly of cholesteryl esters into CMs and VLDL \\
\hline MTP inhibition & Impaired assembly and secretion of apoB lipoproteins by intestine and liver \\
\hline
\end{tabular}

MGAT2, acyl-CoA:monoacylglycerol acyltransferase-2. and ref. 68). Accordingly, knockout of apoA-I (68) or its receptor, Abca1 (69), impairs apoB-independent cholesterol absorption. The role of NPC1L1 in this process has not yet been examined, but it is unlikely that shuttling of UC into the ER and then esterification by ACAT2 would be required (Figure 2). Absorption of mediumchain triglycerides also occurs independently of apoB. The lipids are hydrolyzed in the gut lumen and absorbed directly into portal blood as nonesterified fatty acids (NEFAs) in a process that may involve apoA-IV (70). These apoB-independent pathways are exploited to treat deficiencies in essential fatty acids and fat-soluble vitamins that develop in patients with abetalipoproteinemia or $\mathrm{CM}$ retention disease. The regimes involve administration of lowfat diets supplemented with medium-chain triglycerides, as well as high oral doses of fat-soluble vitamins that the intestine can then incorporate into lipoproteins resembling HDL. Lastly, proteins other than apoB mediate each step in the reabsorption of bile acids by the terminal ileum. Efficient transport of bile acids from the gut lumen into ileal enterocytes occurs via the apical $\mathrm{Na}^{+}$-dependent bile acid transporter and via $\mathrm{Na}^{+}$-independent organic anion transporting peptides (71). Clinically available bile acid sequestrants, as well as new investigational agents that inhibit bile acid transporters, deplete the enterohepatic circulation of bile acids by blocking ileal reabsorption. In response, the liver increases bile acid synthesis, a process that consumes hepatic cholesterol, stimulates LDL receptor expression, and thereby lowers plasma LDL levels.

\section{CM catabolism: branch point between storage and combustion}

\section{Regulated hydrolysis of CM triglycerides}

Enzymatically active dimers of LpL bound to the capillary endothelium of adipose tissue and striated muscle hydrolyze the triglycerides of CMs and VLDL, releasing NEFAs for local and systemic use (Figure 1; reviewed in refs. 72, 73). Hydrolysis by LPL converts CMs into smaller, cholesteryl ester-rich remnant lipoproteins that are released back into the bloodstream. Studies in vitro established heparan sulfate proteoglycans (HSPGs) on the surface of cultured endothelial cells as tethering molecules for LpL (72). Each HSPG molecule consists of a protein core onto which the cell has assembled highly negatively charged sugar polymers, called heparan sulfate, that bind positively charged domains on numerous proteins, including LpL (for a brief primer on proteoglycan biology, see ref. 74). Mutations in LpL and its cofactor, apoC-II, are responsible for certain rare familial chylomicronemias, confirming in humans that these molecules are rate limiting in CM catabolism $(73,75)$. Human polymorphisms of $2 \mathrm{LpL}$ inhibitors, apoC-III and apoA-II, correlate with altered postprandial lipoprotein metabolism (43). Capillary endothelium does not make LpL, but instead picks it up from underlying adipocytes and striated muscle cells (73). Studies in vitro indicate that transcytosis of the enzyme for display on the luminal side is mediated by basolateral HSPGs acting in concert with the VLDL receptor (Figure 3 and ref. 76).

Because $\mathrm{LpL}$ is rate limiting, this system provides a crucial metabolic branch point between storage of CM calories in adipose tissue and combustion in striated muscle (Figures 1 and 3). Targeted overexpression of $\mathrm{LpL}$ in muscle prevents diet-induced obesity in mice, apparently by diverting CM lipolysis away from fat and into muscle (77). Likewise, a targeted deficiency of LPL in adipose tissue ameliorates obesity in hyperphagic mice (2). Formal energy balance studies in these mice would be of interest. Similar phenomena occur physiologically in humans: differential regulation of $\mathrm{LpL}$ activity in fat versus striated muscle diverts triglycerides to adipose tissue in the fed and sedentary states, but to muscle during fasting $(3,78)$ or exercise (79) (Figure 1). These molecular processes likely contribute to the strong correlation between obesity and the tendency to sit $(79,80)$ and overeat (81) (Table 1). Published data on the relevant points of control show considerable complexity (3) and include regulation of $L P L$ mRNA transcription, LPL mRNA stability, LpL protein synthesis, maturation in the ER (82), glycosylation, posttranslational degradation of LpL via its binding to HSPGs on the surface of adipocytes (83) and myocytes, and posttranslational disruption of enzymatically active LpL dimers by angiopoietin-like proteins $(47,84)$, which are themselves regulated by feeding, fasting, nuclear receptor ligands, and gut microbiota (Table 1 and refs. $84,85)$. Despite the regulation of LpL, NEFAs can be delivered to skeletal muscle in excess of its capacity for $\beta$-oxidation, particularly in the context of obesity and physical inactivity (86). Subsequent accumulation of triglycerides within myocytes may cause insulin resistance $(86,87)$, a situation that can be corrected in animals by exercise (Table 1 and ref. 88).

\section{A conceptual difficulty, solved by 2 unexpected participants}

The liver also contains HSPGs and, in the fed state, LpL (reviewed in refs. 39, 40). This presents a conceptual difficulty: How do CMs avoid hepatic clearance before their conversion into remnant 


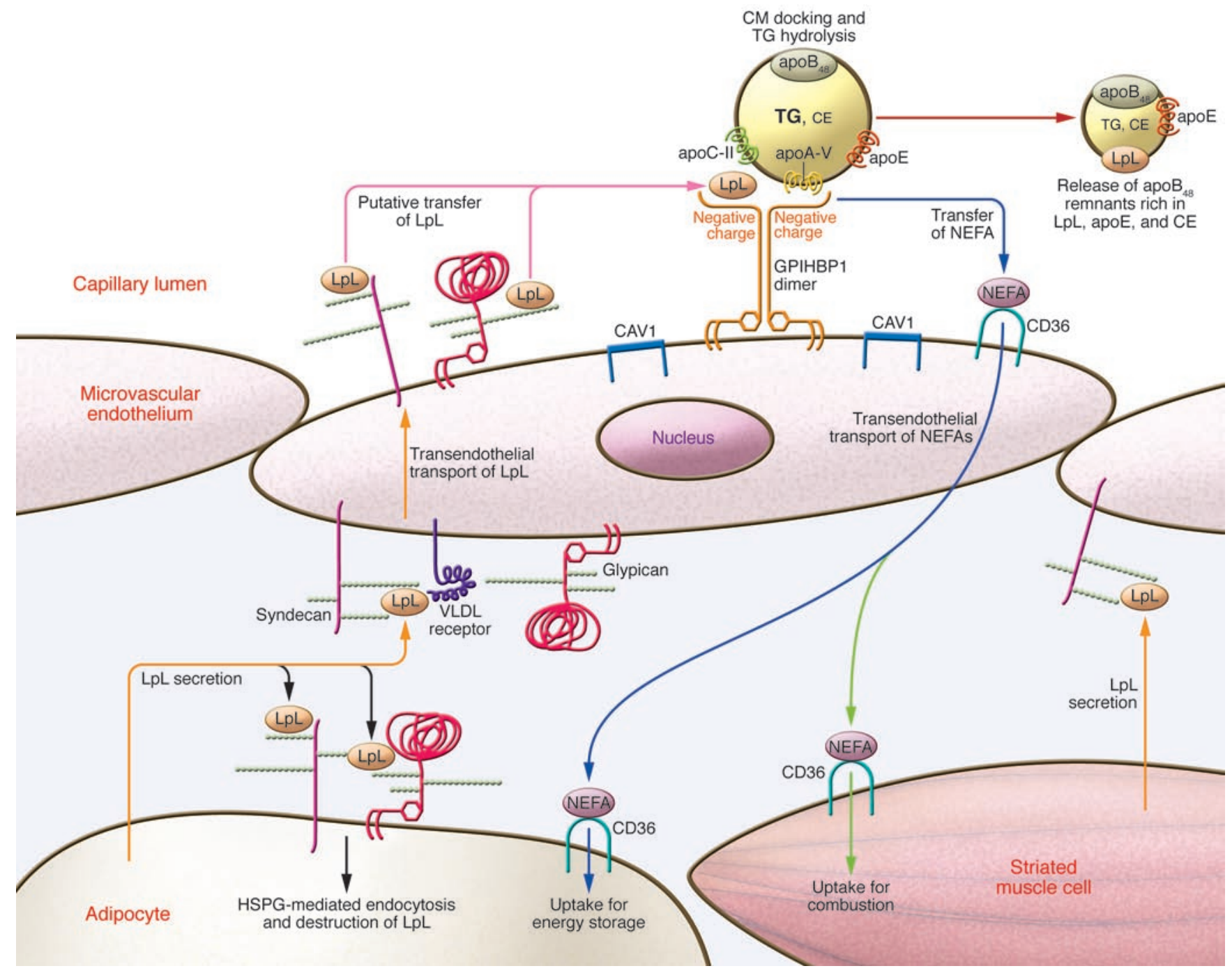

Figure 3

Integrated model of CM binding and hydrolysis on peripheral capillary endothelium. Adipose tissue and striated muscle each synthesize LpL, regulated by the fasted/fed and active/sedentary metabolic states. HSPGs on the surfaces of these cells capture and internalize LpL for degradation. LpL that escapes degradation will be picked up by HSPGs and VLDL receptors on the basal surface of overlying endothelial cells for transcytosis to the luminal surface of capillaries (orange arrows). Heparan sulfate side-chains of syndecan and glypican are denoted by chains of small spheres. The major HSPGs of endothelium, syndecans and glypicans, move into detergent-insoluble membrane microdomains (rafts) rich in caveolin-1 (CAV1) upon clustering. On the apical surface, they encounter GPIHBP1, which should also move into rafts upon clustering. The highly negatively charged N-terminal domain of GPIHBP1 binds LpL with approximately 10 -fold greater affinity than do endothelial HSPGs. Thus, after transcytosis, LpL should be torn away from syndecans and glypicans onto GPIHBP1 (pink arrows). Dimers of GPIHBP1 bind LpL and $\mathrm{CMs}$, thereby providing a platform for $\mathrm{CM}$ docking and triglyceride lipolysis. These processes are facilitated by apoC-II and apoA-V. Lipolysis generates NEFAs that are transported by another raft molecule, CD36, across the endothelium and into adipocytes for energy storage (blue arrows) or into striated myocytes for combustion (green arrows). After hydrolysis of $\mathrm{CM}_{\text {triglycerides, the endothelium releases apoB }} \mathrm{B}_{8}$ remnant lipoproteins that are rich in LpL, apoE, and cholesteryl ester back into the circulation (red arrow). Under normal circumstances, these remnant particles undergo safe, swift uptake by the liver.

lipoproteins? In fact, the heparan sulfate chains in liver exhibit extreme structures of high flexibility and negative charge (89), and hence show far stronger ligand affinity than does heparan sulfate in other tissues, including peripheral endothelium. Thus, injection of $\mathrm{LpL}$ or other heparin-binding proteins results in primarily hepatic clearance, with little if any delivery into fat or muscle. Direct hepatic uptake of CMs may be diminished by their secretion from the intestine into lymph, which bypasses the portal circulation (Figure 1). A common explanation holds that hepatic uptake of CMs can occur only after these particles are shrunk by extrahepatic lipolysis into remnant lipoproteins that then become small enough to squeeze through the approximately $100-\mathrm{nm}$ fenestrae in the endothelial lining of liver sinusoids. Yet most of the triglyceride-rich lipoproteins in plasma from hyperchylomicronemic mice were reported to be less than $100 \mathrm{~nm}$ in diameter (90). Thus, there must be a molecular, not just a physical, explanation for the robust initial targeting of these particles to adipose tissue and striated muscle under normal conditions. 
The answer appears to reside in the recent discoveries of 2 unsuspected participants in peripheral lipolysis: apoA-V on lipoproteins and the glycosylphosphatidylinositol-anchored (GPIanchored) HDL-binding protein 1 (GPIHBP1) on peripheral endothelium. Adipocyte-specific knockout of a third molecule, LDL receptor-related protein-1 (Lrp1), revealed a role in lipid uptake from model CM remnants into brown fat, but not into white fat (91), and so its relevance to human physiology remains unclear at this point.

$A P O A 5$. In 2001, 2 laboratories discovered a new apoprotein gene (dubbed APOA5, within the well-known APOA1/APOC3/APOA4 gene cluster) using a computational search for conserved genomic regions and a survey of upregulated genes during liver injury; the protein product is referred to as apoA-V or APOA5. To demonstrate its relevance to lipoprotein physiology, Pennacchio et al. reported that mice transgenic for human apoA-V showed a two-thirds reduction in plasma triglyceride levels; that Apoa5 knockout mice exhibited 4 times the plasma triglyceride concentrations of control animals; and that human polymorphisms were significantly associated with plasma triglyceride values (92). Subsequent studies confirmed the link between human apoA- $V$ variants and both fasting and postprandial hypertriglyceridemia (43, 47, 75, 93). Despite its substantial effects, apoA-V exhibits surprisingly low levels in normal plasma compared with other apoproteins (approximately $180 \mathrm{ng} / \mathrm{ml}$, about $0.02 \%$ the level of apoA-I), which is why it was missed in earlier compositional studies of lipoproteins. The normal apoA-V protein potently facilitates LpL-mediated triglyceride hydrolysis in animals (94) and in humans (93). In addition, apoA-V facilitates hydrolysis of triglyceride-rich lipoproteins by $\mathrm{LPL}$ in solution or bound to HSPGs on the surface of cultured cells (94). This last finding requires revisiting based on recent results concerning the second molecule, GPIHBP1 (Figure 3).

GPIHBP1. During a screen of hundreds of knockout mice at Genentech, overly milky lipemia was unexpectedly noted in mice lacking Gpihbp1 (90, 95), a GPI-anchored membrane protein originally identified by its ability to bind HDL (96). Subse-

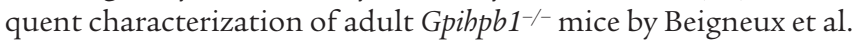
indicated high plasma concentrations of large, triglyceride-rich $\mathrm{apoB}_{48}$ lipoproteins in the CM/VLDL size range, low plasma levels of HDL, and impaired CM removal (apparently owing to defective lipolysis in peripheral tissues), but only about $40 \%$ reduction in postheparin plasma LpL levels (90).

Mechanistic investigations of this phenotype led to the hypothesis that Gpihbp1, not HSPGs, provides the key platform for LpL-mediated lipolysis of CM triglycerides on microvascular endothelium (see "Integrated model for CM hydrolysis and local NEFA uptake"; Figure 3; and refs. 90, 95). Expression of Gpihbp1 in vivo was found exclusively on the luminal surface of endothelial cells, with particularly high levels in the capillaries of adipose tissue and striated muscle (90). Consistent with a role in caloric delivery, Gpibbp1 mRNA levels in muscle were doubled by fasting (90), reminiscent of the metabolic control of LpL discussed above (Table 1). Levels of Gpihbp1 in liver are low, which likely contributes to poor hepatic uptake of CMs until triglyceride hydrolysis transforms them into remnant lipoproteins that display different binding epitopes.

In vitro, the transfection of HSPG-deficient cells with a Gpihbp1 expression construct caused robust, high-affinity binding of LpL $\left(K_{\mathrm{d}}, 36 \mathrm{nM}\right.$; ref. 90). For comparison, LpL binding to the HSPGs on cultured endothelial cells exhibits a $K_{\mathrm{d}}$ of $140-520 \mathrm{nM}(72)$, which implies that Gpihbp1 could tear LpL away from endothelial
HSPGs after HSPG-mediated transcytosis (Figure 3). Transfection of LDL receptor-deficient cells with a Gpihbp1 expression vector increased their binding to artificial apoA-V/phosphatidylcholine disks and to CMs harvested from Gpibbp $p^{-1-}$ mice. In each case, the interactions appear to be electrostatic: LpL, apoA-V, and other CM apoproteins contain positively charged domains, and Gpihbp1 contains a highly negatively charged region toward its $\mathrm{N}$ terminus, far from the plasma membrane. By binding both LpL and CMs, Gpihpb1 may bring them physically together to facilitate lipolysis. If LpL and CMs occupy the same negatively charged site on Gpihbp1, efficient lipolysis could require dimerization of Gpihbp1, a process that may be facilitated by its Ly-6 domain (90).

The Gpibbp1 $1^{-/-}$mouse is the second of only 2 examples of chylomicronemia related to a defect in the endothelium of adipose tissue and muscle $(90,97)$. Interestingly, a rare mutation in GPIHBP1 has been associated with human chylomicronemia, although this variant appears to function normally in vitro (98). Further studies are needed in order to definitively establish the role of GPIHBP1 in human physiology, although it presumably serves functions similar to those of its murine homolog.

\section{Integrated model for CM hydrolysis and local NEFA uptake}

The known properties of these molecular participants suggest that CM hydrolysis and local NEFA uptake are facilitated by being concentrated within cholesterol-rich membrane microdomains (rafts) along the luminal endothelial plasma membrane (Figure 3). The major HSPGs expressed by endothelial cells include the syndecan family of transmembrane HSPGs and the glypican family of GPI-anchored HSPGs. We (99) and others (100) showed that syndecan HSPGs move into rafts upon clustering. Binding just a dimer, such as enzymatically active LpL, appears sufficient for syndecan to localize into rafts (99). Importantly, GPI-anchored proteins also move laterally into rafts upon clustering (101), and so I expect that GPIHBP1 and glypican HSPGs would colocalize with syndecans after transendothelial transport of active, dimeric $\mathrm{LpL}$. Close proximity should facilitate the transfer of $\mathrm{LpL}$ from syndecan and glypican HSPGs onto higher-affinity binding sites on nearby GPIHBP1 (Figure 3).

Efficient importation of newly released NEFAs by fat and muscle relies on CD36, a member of the class B family of scavenger receptors that is expressed by adipocytes, myocytes, and microvascular endothelial cells. Adipose expression of Cd36 mRNA is induced in vivo by high-fat feeding, apparently to facilitate fatty acid uptake and storage (102). Adipose expression is also increased in human type 2 diabetes (103), which likely accelerates the delivery of NEFA calories into this tissue and could worsen obesity (Table 1). Importantly, the Cd36 fatty acid translocase localizes to and requires rafts for long-chain fatty acid uptake into adipocytes (104) and other cells, and I speculate the same is true for transendothelial NEFA transport (Figure 3). Again, close proximity would facilitate molecular transfer, in this case, of NEFAs from LpL-GPIHBP1-apoA-V/CM complexes onto CD36. Consistent with this model, disruption of the murine gene for caveolin-1, a scaffolding protein that is highly expressed in endothelial cells and adipocytes and directs the formation of a specific class of cholesterol-rich membrane microdomains, impairs lipolysis of postprandial lipoproteins despite normal heparin-releasable LpL activity (97). The caveolin-1 knockout also interferes with the postprandial importation of NEFAs across endothelium into adipose tissue and thereby blocks the development of diet-induced 


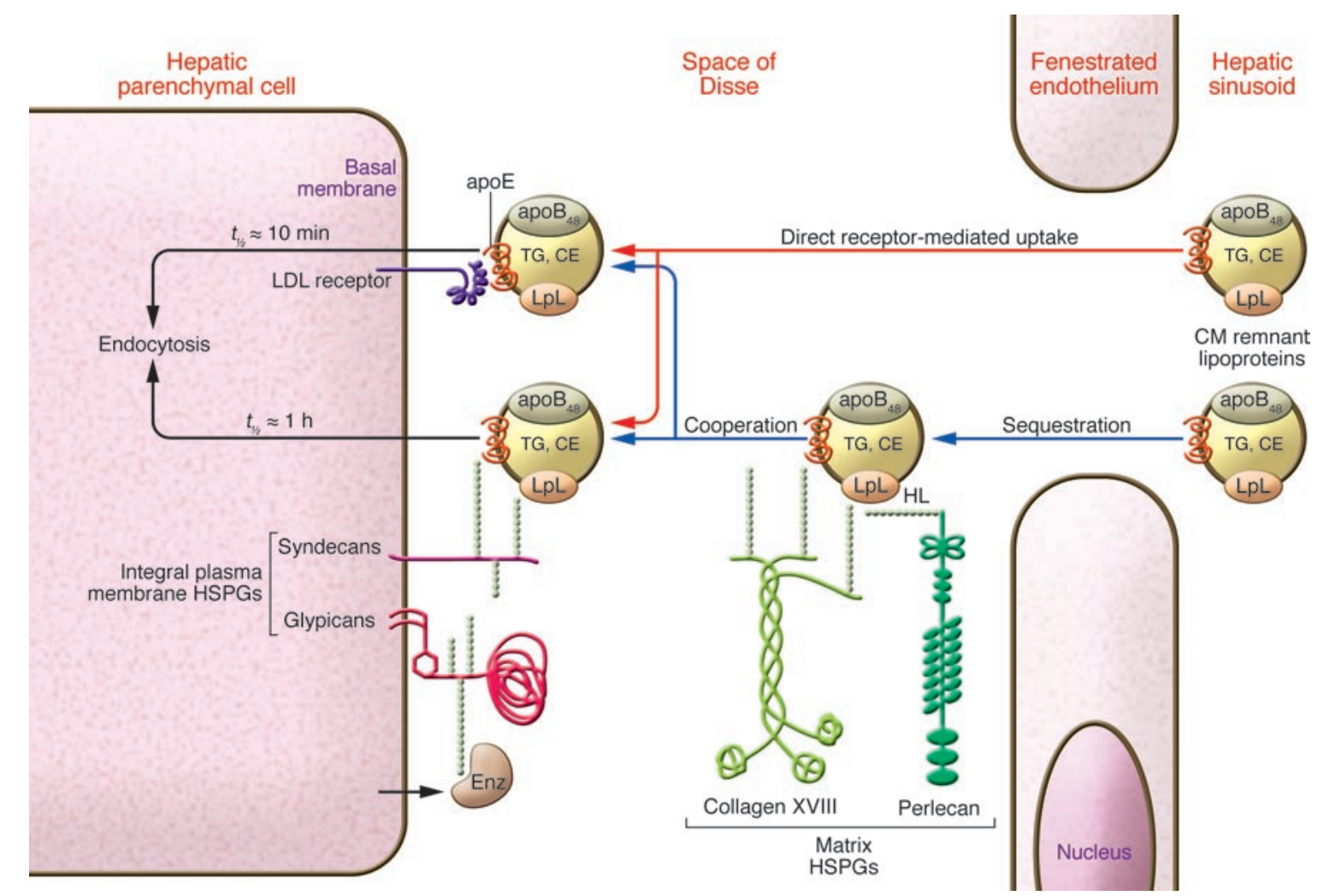

Figure 4

Efficient hepatic uptake of CM remnant lipoproteins. Hepatic HSPGs exhibit extreme structural features of their carbohydrate side-chains that enhance ligand binding. Thus, HSPGs in the liver rapidly pull $\mathrm{apoB}_{48}$ remnant lipoproteins out of plasma via interactions with positively charged proteins on the particles, chiefly LpL and apoE. Once in the liver, the particles encounter hepatic lipase (HL), which serves as an additional bridging molecule between HSPGs and lipoproteins. Shown are 2 pathways for particle clearance. The first is direct receptor-mediated uptake, in which cholesteryl ester-rich $a_{0} B_{48}$ remnant lipoproteins pass from the hepatic sinusoid through the fenestrated endothelium and then bind directly to integral plasma membrane receptors (red arrows; shown are the LDL receptor, which binds apoE, and the syndecan and glypican HSPGs, which bind LpL, hepatic lipase, and apoE). The second clearance mechanism is a cooperative pathway, in which apoB ${ }_{48}$ remnant lipoproteins from the hepatic sinusoid are first sequestered by matrix HSPGs within the space of Disse and then taken up in cooperation with the integral plasma membrane receptors (blue arrows; shown are the collagen XVIII and perlecan HSPGs). Also shown is one of several secreted enzymes (Enz), such as heparanase or the heparan 6-O-endosulfatases, that are expressed by liver, degrade HSPGs, and may dampen these processes.

obesity (97). Likewise, mutation of human caveolin-1 is a cause of dyslipidemia with lipodystrophy, which suggests that the same processes occur in people (105).

\section{CM catabolism: rapid hepatic uptake of apoB $_{48}$ remnants}

Over a quarter century ago, astute observations in vivo revealed that efficient hepatic uptake of remnant lipoproteins from plasma does not require LDL receptors $(46,106-108)$. This insight launched a long, difficult search for the molecules responsible for this crucial pathway. Over many years, several protein candidates were identified, most notably LRP1 (109), but disablement of hepatic LRP1 in vivo fails to result in poor clearance of remnant lipoproteins, particularly when LDL receptors are present, as they are in normal human physiology (109-112). Instead, work from our laboratory (39) and others $(44,45,111,113,114)$ implicated HSPGs, contradicting a common view that these molecules play only passive, structural roles (Figure 4 and refs. 40,74). Extreme structural features of hepatic HSPGs that enhance ligand affinity (89), noted above, direct remnants into the liver. Moreover, strong evidence now exists for a physiologic role for HSPGs in remnant lipoprotein clearance in humans $(44,45)$.
Because many HSPG core proteins are highly conserved, yet dissimilar from each other, we hypothesized that different classes of HSPGs would mediate distinct endocytic pathways (Figure 4 and refs. 40, 74). To date, we have demonstrated a specific endocytic pathway mediated by syndecan HSPGs when they become clustered $(40,99)$ and a separate pathway with distinct subcellular trafficking mediated by the perlecan HSPG when it is adherent to the cell surface $(74,115,116)$. Endocytosis via glypican HSPGs has also been reported (117).

Normal liver expresses several syndecans, the perlecan HSPG, glypicans, the long variant of collagen XVIII, and agrin (Figure 4 and refs. 118-120). The contribution of each of these HSPGs to remnant lipoprotein uptake in vivo needs to be evaluated. Because syndecan-1 is particularly abundant on the sinusoidal surface of hepatic parenchymal cells (118), it is a strong candidate to participate in remnant lipoprotein clearance in vivo (40). In this context, we proposed that hepatic HSPGs can be divided into 2 functional subsets (39): those that cells readily internalize, especially the integral plasma membrane syndecans (40) and glypicans (117), and those that cells might not readily internalize, meaning extracellular HSPGs of the basement membrane and other matrix, such as collagen XVIII, agrin, and 
most of the perlecan within the liver. Thus, in our model, cell surface syndecan and glypican HSPGs bind, then directly carry, remnant lipoproteins and other ligands into the cells, whereas matrix HSPGs bind and sequester their ligands; however, endocytosis requires cooperation with cell surface receptors, particularly syndecan-1, the LDL receptor (115), and possibly glypicans (Figure 4). Because syndecan-mediated endocytosis proceeds less rapidly $\left(t_{1 / 2}\right.$, about $1 \mathrm{~h}$; ref. $40)$ than uptake via LDL receptors $\left(t_{1 / 2}\right.$, about $\left.10 \mathrm{~min}\right)$, this model may account for quick hepatic uptake of remnants from plasma into the space of Disse, followed by continued internalization into hepatocytes even when LDL receptors are absent, although the internalization occurs at a reduced speed (Figure 4 and refs. 46, 108).

Diabetes mellitus and related conditions have been reported to cause CM overproduction $(15,67)$, delayed hydrolysis of CM triglyceride in the periphery, and - perhaps most important to the development of vascular disease - substantial impairment in hepatic clearance of atherogenic CM remnants $(4,13,67,113$, 121). Persistent remnants, unlike CMs themselves, readily enter the arterial wall and are retained there (4), initiating and accelerating atherosclerosis $(6,11,12,122)$. Impaired hepatic clearance of $\mathrm{apoB}_{48}$ remnants from plasma arises in large part from the failure of diabetic liver to properly assemble HSPGs (113, 122-124). Specific molecular mediators of defective hepatic HPSG assembly in diabetes include suppression of heparan sulfate $\mathrm{N}$-deacetylase $/ \mathrm{N}$ sulfotransferase-1 (Ndst1) (122), a key enzyme in the biosynthesis of highly charged, flexible heparan sulfate chains that avidly bind ligands, and suppression of Fxr (125), an upstream regulator of syndecan-1 expression (126). Liver-specific knockout of the same heparan sulfate assembly enzyme, $\mathrm{Ndst} 1$, produces hypertriglyceridemia and a robust delay in the clearance of postprandial remnant lipoproteins (111), an important observation that confirms the role of HSPGs as remnant receptors (39) and the identification of Ndst1 as a key regulatory factor (Table 1 and ref. 122). Regarding reversibility, moderate caloric restriction of obese, type 2 diabetic mice corrects their defect in remnant lipoprotein clearance (127), and similar effects occur in human diabetic subjects after short-term weight loss (128). This body of work provides tremendous explanatory power, elucidating the normal physiologic uptake of remnant lipoproteins by the liver as well as the unhealthy persistence of these particles in diabetes and related syndromes (Figure 4).

Finally, it has been hypothesized that molecules other than HSPGs and LDL receptor family members may also contribute to hepatic clearance of remnants, but their nature remains a matter of speculation (112). One candidate is scavenger receptor class B type I (Srb1), proposed as a docking molecule in the liver for CM remnants (129). Because conflicting literature indicates that the hepatic expression of SRB1 may be up- or downregulated in diabetes, its contribution to poor removal of remnants in that condition remains unknown.

\section{Conclusions and future directions}

The central question facing the field is how this explosion in knowledge might benefit human health. Despite our extensive molecular understanding, major health problems related to intestinal absorption of lipids and other overly abundant macronutrients - coupled with physical inactivity - continue to grow worldwide, particularly atherosclerosis, obesity, and type 2 diabetes. Moreover, as noted throughout this review, many derangements in lipid absorption and trafficking can be reversed by well-known lifestyle changes and available pharmaceutical agents.

The situation could be improved by 2 general approaches. The first is better implementation of recognized strategies. Improvements in lifestyle can be facilitated by education and counseling, but also by pancreatic lipase inhibitors (Table 2), novel appetite suppressants, and surgical techniques that reduce food intake and body weight. New methods of lowering plasma concentrations of atherogenic apoB-containing lipoproteins are also under development $(11,12)$. Public policy, which is extensively reviewed elsewhere $(80,81)$, remains crucial to promoting active living and the cultivation and consumption of affordable, healthy foods.

The second approach is the exploration of new therapeutic targets. Despite its importance, postprandial dyslipidemia has often been overlooked because it remains inconvenient to accurately assess. Improved $\mathrm{apoB}_{48}$-specific assays will likely help. Moreover, nonfasting plasma triglyceride levels, an easy yet nonstandardized measurement related to postprandial dyslipidemia, correlate with future cardiovascular events and could serve as a therapeutic target $(4,13)$. Novel points of control could be found through additional molecular characterizations of fed/sedentary states as well as characterization of the beneficial changes that occur during caloric restriction and exercise. For example, reversal of intestinal insulin resistance has not been explored; the molecular basis for ezetimibe-resistant cholesterol absorption needs elucidation; metabolic regulation of GPIHBP1 and its role in human biology require further study; and improvements in hepatic clearance of remnant lipoproteins upon weight loss remain completely uncharacterized on a molecular level.

There is no substitute for a combined approach. The exciting opportunities outlined above for fundamental and translational advances in the biology of lipid absorption and transport will require an aggressive mix of new basic, clinical, and public policy efforts.

\section{Acknowledgments}

The author acknowledges support from the American Heart Association, the American Diabetes Association, the Ruth and Yonatan BenAvraham Fund, and 2 awards from the NIH for his work on HSPGs (R01HL38956, 1994-1999; R01HL73898, 2005-2009). The author thanks Edward A. Fisher for his critical review of the manuscript.

Address correspondence to: Kevin Jon Williams, Division of Endocrinology, Diabetes, and Metabolic Diseases, Thomas Jefferson University, Curtis 320B, 1015 Walnut Street, Philadelphia, Pennsylvania 19107-5005, USA. Phone: (215) 503-1272; Fax: (215) 923-7932; E-mail: K_Williams@mail.jci.tju.edu.

\footnotetext{
1. Konstantinov, I.E., Mejevoi, N., and Anichkov, N.M. 2006. Nikolai N. Anichkov and his theory of atherosclerosis. Tex. Heart Inst. J. 33:417-423.

2. Weinstock, P.H., et al. 1997. Lipoprotein lipase controls fatty acid entry into adipose tissue, but fat mass is preserved by endogenous synthesis in mice deficient in adipose tissue lipoprotein lipase. Proc. Natl. Acad. Sci. U. S. A. 94:10261-10266.

3. Mead, J.R., Irvine, S.A., and Ramji, D.P. 2002. Lipoprotein lipase: structure, function, regulation, and
}

role in disease. J. Mol. Med. 80:753-769.

4. Nordestgaard, B.G., Benn, M., Schnohr, P., and Tybjaerg-Hansen, A. 2007. Nonfasting triglycerides and risk of myocardial infarction, ischemic heart disease, and death in men and women. JAMA. 298:299-308.

5. Flood, C., et al. 2002. Identification of the proteoglycan binding site in apolipoprotein B48. J. Biol. Chem. 277:32228-32233.

6. Williams, K.J., and Tabas, I. 1995. The response-to- retention hypothesis of early atherogenesis. Arterioscler. Thromb. Vasc. Biol. 15:551-561.

7. Karpe, F., and Hamsten, A. 1995. Postprandial lipoprotein metabolism and atherosclerosis. Curr. Opin. Lipidol. 6:123-129.

8. Adiels, M., Olofsson, S.-O., Taskinen, M.-R., and Borén, J. 2008. Overproduction of very low-density lipoproteins is the hallmark of the dyslipidemia in the metabolic syndrome. Arterioscler. Thromb. Vasc. Biol. 28:1225-1236. 
9. Skålén, K., et al. 2002. Subendothelial retention of atherogenic lipoproteins in early atherosclerosis. Nature. 417:750-754.

10. Nakashima, Y., Fujii, H., Sumiyoshi, S., Wight, T.N., and Sueishi, K. 2007. Early human atherosclerosis: accumulation of lipid and proteoglycans in intimal thickenings followed by macrophage infiltration. Arterioscler. Thromb. Vasc. Biol. 27:1159-1165.

11. Tabas, I., Williams, K.J., and Borén, J. 2007. Subendothelial lipoprotein retention as the initiating process in atherosclerosis: update and therapeutic implications. Circulation. 116:1832-1844.

12. Williams, K.J., Feig, J.E., and Fisher, E.A. 2008. Rapid regression of atherosclerosis: insights from the clinical and experimental literature. Nat. Clin. Pract. Cardiovasc. Med. 5:91-102.

13. Bansal, S., et al. 2007. Fasting compared with nonfasting triglycerides and risk of cardiovascular events in women. JAMA. 298:309-316.

14. Gaziano, T.A. 2005. Cardiovascular disease in the developing world and its cost-effective management. Circulation. 112:3547-3553.

15. Federico, L.M., Naples, M., Taylor, D., and Adeli, K. 2006. Intestinal insulin resistance and aberrant production of apolipoprotein B48 lipoproteins in an animal model of insulin resistance and metabolic dyslipidemia: evidence for activation of protein tyrosine phosphatase-1B, extracellular signal-related kinase, and sterol regulatory elementbinding protein-1 $\mathrm{c}$ in the fructose-fed hamster intestine. Diabetes. 55:1316-1326.

16. Clader,J.W. 2004. The discovery of ezetimibe: a view from outside the receptor. J. Med. Chem. 47:1-9.

17. Garcia-Calvo, M., et al. 2005. The target of ezetimibe is Niemann-Pick C1-Like 1 (NPC1L1). Proc. Natl. Acad. Sci. U. S. A. 102:8132-8137.

18. Huff, M.W., Pollex, R.L., and Hegele, R.A. 2006. NPC1L1: Evolution from pharmacological target to physiological sterol transporter. Arterioscler. Thromb. Vasc. Biol. 26:2433-2438.

19. Altmann, S.W., et al. 2004. Niemann-Pick C1 Like 1 protein is critical for intestinal cholesterol absorption. Science. 303:1201-1204.

20. Davies, J.P., Scott, C., Oishi, K., Liapis, A., and Ioannou, Y.A. 2005. Inactivation of NPC1L1 causes multiple lipid transport defects and protects against diet-induced hypercholesterolemia. J. Biol. Chem. 280:12710-12720.

21. Fahmi, S., Yang, C., Esmail, S., Hobbs, H.H., and Cohen, J.C. 2008. Functional characterization of genetic variants in NPC1L1 supports the sequencing extremes strategy to identify complex trait genes. Hum. Mol. Genet. 17:2101-2107.

22. Yu, L., et al. 2006. Cholesterol-regulated translocation of NPC1L1 to the cell surface facilitates free cholesterol uptake. J. Biol. Chem. 281:6616-6624.

23. Knopfel, M., et al. 2007. Multiple plasma membrane receptors but not NPC1L1 mediate high-affinity, ezetimibe-sensitive cholesterol uptake into the intestinal brush border membrane. Biochim. Biophys. Acta. 1771:1140-1147.

24. Field, F.J., Watt, K., and Mathur, S.N. 2007. Ezetimibe interferes with cholesterol trafficking from the plasma membrane to the endoplasmic reticulum in CaCo-2 cells. J. Lipid Res. 48:1735-1745.

25. Temel, R.E., et al. 2007. Hepatic Niemann-Pick C1-like 1 regulates biliary cholesterol concentration and is a target of ezetimibe. J. Clin. Invest. 117:1968-1978.

26. Briand, F., Millar, J., Billheimer, J., Rothblat, G., and Rader, D. 2007. Inhibition of cholesterol absorption with ezetimibe promotes macrophage to feces reverse cholesterol transport in mice [abstract]. Circulation. II-159-II-160.

27. Sehayek, E., and Hazen, S.L. 2008. Cholesterol absorption from the intestine is a major determinant of reverse cholesterol transport from peripheral tissue macrophages. Arterioscler. Thromb. Vasc.
Biol. 28:1296-1297.

28. Lally, S., Owens, D., and Tomkin, G.H. 2007. Genes that affect cholesterol synthesis, cholesterol absorption, and chylomicron assembly: the relationship between the liver and intestine in control and streptozotosin diabetic rats. Metabolism. 56:430-438.

29. Gylling, H., et al. 2007. Markers of absorption and synthesis of cholesterol in men with type 1 diabetes. Diabetes Metab. Res. Rev. 23:372-377.

30. Lally, S., Tan, C.Y., Owens, D., and Tomkin, G.H. 2006. Messenger RNA levels of genes involved in dysregulation of postprandial lipoproteins in type 2 diabetes: the role of Niemann-Pick C1-like 1, ATP-binding cassette, transporters G5 and G8, and of microsomal triglyceride transfer protein. Diabetologia. 49:1008-1016.

31. Gylling, H., and Miettinen, T.A. 1996. Cholesterol absorption and lipoprotein metabolism in type II diabetes mellitus with and without coronary artery disease. Atherosclerosis. 126:325-332.

32. Davis, H.R., Jr., et al. 2007. Deficiency of NiemannPick C1 like 1 prevents atherosclerosis in apoE ${ }^{-/}$ mice. Arterioscler. Thromb. Vasc. Biol. 27:841-849.

33. Kastelein, J.J., et al. 2008. Simvastatin with or without ezetimibe in familial hypercholesterolemia. N. Engl. J. Med. 358:1431-1443.

34. Yu, L., et al. 2002. Disruption of Abcg5 and Abcg8 in mice reveals their crucial role in biliary cholesterol secretion. Proc. Natl. Acad. Sci. U. S. A. 99:16237-16242.

35. Graf, G.A., et al. 2003. ABCG5 and ABCG8 are obligate heterodimers for protein trafficking and biliary cholesterol excretion. J. Biol. Chem. 278:48275-48282.

36. Yu, L., et al. 2003. Stimulation of cholesterol excretion by the liver $\mathrm{X}$ receptor agonist requires ATPbinding cassette transporters G5 and G8. J. Biol. Chem. 278:15565-15570.

37. Pulai, J.I., et al. 1997. Normal intestinal dietary fat and cholesterol absorption, intestinal apolipoprotein B (apoB) mRNA levels, and apoB48 synthesis in a hypobetalipoproteinemic kindred without any apoB truncation. Metabolism. 46:1095-1100

38. Felts, J.M., Itakura, H., and Crane, R.T. 1975. The mechanims of assimilation of consitutents of chylomicrons, very low density lipoproteins and remnants - a new theory. Biochem. Biophys. Res. Commun. 66:1467-1475.

39. Williams, K.J., et al. 1992. Mechanisms by which lipoprotein lipase alters cellular metabolism of lipoprotein(a), low density lipoprotein, and nascent lipoproteins. Roles for low density lipoprotein receptors and heparan sulfate proteoglycans. J. Biol. Chem. 267:13284-13292.

40. Fuki, I.V., et al. 1997. The syndecan family of proteoglycans: novel receptors mediating internalization of atherogenic lipoproteins in vitro. J. Clin. Invest. 100:1611-1622.

41. Zheng, C., Murdoch, S.J., Brunzell, J.D., and Sacks, F.M. 2006. Lipoprotein lipase bound to apolipoprotein B lipoproteins accelerates clearance of postprandial lipoproteins in humans. Arterioscler. Thromb. Vasc. Biol. 26:891-896.

42. Zambon, A., Deeb, S.S., Bensadoun, A., Foster, K.E., and Brunzell, J.D. 2000. In vivo evidence of a role for hepatic lipase in human apoB-containing lipoprotein metabolism, independent of its lipolytic activity. J. Lipid Res. 41:2094-2099.

43. López-Miranda, J., et al. 2006. Postprandial lipoprotein metabolism, genes and risk of cardiovascular disease. Curr. Opin. Lipidol. 17:132-138.

44. Ji, Z.S., Fazio, S., and Mahley, R.W. 1994. Variable heparan sulfate proteoglycan binding of apolipoprotein $\mathrm{E}$ variants may modulate the expression of type III hyperlipoproteinemia. J. Biol. Chem. 269:13421-13428.
45. Mann, W.A., et al. 1995. Apolipoprotein E isoforms and rare mutations: parallel reduction in binding to cells and to heparin reflects severity of associated type III hyperlipoproteinemia. J. Lipid Res. 36:517-525.

46. Mortimer, B.C., Beveridge, D.J., Martins, I.J., and Redgrave, T.G. 1995. Intracellular localization and metabolism of chylomicron remnants in the livers of low density lipoprotein receptor-deficient mice and apoE-deficient mice. Evidence for slow metabolism via an alternative apoE-dependent pathway. J. Biol. Chem. 270:28767-28776.

47. Lusis, A.J., and Pajukanta, P. 2008. A treasure trove for lipoprotein biology. Nat. Genet. 40:129-130.

48. Lo, C.M., et al. 2008. Why does the gut choose apolipoprotein B48 but not B100 for chylomicron formation? Am. J. Physiol. 294:G344-G352.

49. Fisher, E.A., and Ginsberg, H.N. 2002. Complexity in the secretory pathway: the assembly and secretion of apolipoprotein B-containing lipoproteins. J. Biol. Chem. 277:17377-17380.

50. Fisher, E.A., et al. 2001. The triple threat to nascent apolipoprotein-B: evidence for multiple, distinct degradative pathways. J. Biol. Chem. 276:27855-27863.

51. Cartwright, I.J., and Higgins, J.A. 2001. Direct evidence for a two-step assembly of apoB48-containing lipoproteins in the lumen of the smooth endoplasmic reticulum of rabbit enterocytes. J. Biol. Chem. 276:48048-48057.

52. Gusarova, V., Brodsky, J.L., and Fisher, E.A. 2003. Apolipoprotein B100 exit from the endoplasmic reticulum (ER) is COPII-dependent, and its lipidation to very low density lipoprotein occurs post-ER. J. Biol. Chem. 278:48051-48058.

53. Stillemark-Billton, P., Beck, C., Borén, J., and Olofsson, S.O. 2005. Relation of the size and intracellular sorting of apoB to the formation of VLDL1 and VLDL2. J. Lipid Res. 46:104-114.

54. Liao, W., and Chan, L. 2000. Apolipoprotein B, a paradigm for proteins regulated by intracellular degradation, does not undergo intracellular degradation in CaCo2 cells. J. Biol. Chem. 275:3950-3956.

55. Wetterau, J.R., et al. 1992. Absence of microsomal triglyceride transfer protein in individuals with abetalipoproteinemia. Science. 258:999-1001.

56. Cuchel, M., et al. 2007. Inhibition of microsomal triglyceride transfer protein in familial hypercholesterolemia. N. Engl. J. Med. 356:148-156.

57. Jiang, X.C., et al. 2005. Phospholipid transfer protein deficiency impairs apolipoprotein-B secretion from hepatocytes by stimulating a proteolytic pathway through a relative deficiency of vitamin $\mathrm{E}$ and an increase in intracellular oxidants. J. Biol. Chem. 280:18336-18340.

58. Pan, X., and Hussain, M.M. 2007. Diurnal regulation of microsomal triglyceride transfer protein and plasma lipid levels. J. Biol. Chem. 34:24707-24719.

59. Pan, M., et al. 2008. Pre-secretory oxidation, aggregation, and autophagic destruction of apolipoprotein-B: a pathway for late-stage quality control. Proc. Natl. Acad. Sci. U. S. A. 105:5862-5867.

60. Williams, K.J., Brocia, R.W., and Fisher, E.A. 1990. The unstirred water layer as a site of control of apolipoprotein B secretion. J. Biol. Chem. 265:16741-16744.

61. Harris, W.S., and Muzio, F. 1993. Fish oil reduces postprandial triglyceride concentrations without accelerating lipid-emulsion removal rates. Am. J. Clin. Nutr. 58:68-74.

62. Magun, A.M., Mish, B., and Glickman, R.M. 1988. Intracellular apoA-I and apoB distribution in rat intestine is altered by lipid feeding. J. Lipid Res. 29:1107-1116

63. Hockey, K.J., Anderson, R.A., Cook, V.R., Hantgan, R.R., and Weinberg, R.B. 2001. Effect of the apolipoprotein A-IV Q360H polymorphism on postprandial plasma triglyceride clearance. J. Lipid 
Res. 42:211-217.

64. Neeli, I., et al. 2007. Liver fatty acid-binding protein initiates budding of pre-chylomicron transport vesicles from intestinal endoplasmic reticulum. J. Biol. Chem. 282:17974-17984.

65. Jones, B., et al. 2003. Mutations in a Sar1 GTPase of COPII vesicles are associated with lipid absorption disorders. Nat. Genet. 34:29-31.

66. Charcosset, M., et al. 2008. Anderson or chylomicron retention disease: Molecular impact of five mutations in the SAR1B gene on the structure and the functionality of Sar1b protein. Mol. Genet. Metab. 93:74-84.

67. Hogue, J.C., et al. 2007. Evidence of increased secretion of apolipoprotein B-48-containing lipoproteins in subjects with type 2 diabetes. J. Lipid Res. 48:1336-1342.

68. Iqbal, J., and Hussain, M.M. 2005. Evidence for multiple complementary pathways for efficient cholesterol absorption in mice. J. Lipid Res. 46:1491-1501.

69. Temel, R.E., et al. 2005. Intestinal cholesterol absorption is substantially reduced in mice deficient in both ABCA1 and ACAT2. J. Lipid Res. 46:2423-2431.

70. Nishimura, M., Seishima, M., Ohashi, H., and Noma, A. 1996. Effects of lipid administration on lymphatic apolipoprotein A-IV and B output and synthesis. Am. J. Physiol. 271:G322-G329.

71. Alrefai, W.A., and Gill, R.K. 2007. Bile acid transporters: structure, function, regulation and pathophysiological implications. Pharm. Res. 24:1803-1823.

72. Olivecrona, T., and Bengtsson-Olivecrona, G. 1987. Lipoprotein lipase from milk - the model enzyme in lipoprotein lipase research. In Lipoprotein lipase. J. Borensztajn, editor. Evener. Chicago, Illinois, USA. $15-58$.

73. Wang, C.-S., Hartsuck, J., and McConathy, W.J. 1992. Structure and functional properties of lipoprotein lipase. Biochim. Biophys. Acta. 1123:1-17.

74. Williams, K.J., and Fuki, I.V. 1997. Cell-surface heparan sulfate proteoglycans: dynamic molecules mediating ligand catabolism. Curr. Opin. Lipidol. 8:253-262.

75. Wang, J., et al. 2007. Resequencing genomic DNA of patients with severe hypertriglyceridemia (MIM 144650). Arterioscler. Thromb. Vasc. Biol. 27:2450-2455.

76. Obunike, J.C., et al. 2001. Transcytosis of lipoprotein lipase across cultured endothelial cells requires both heparan sulfate proteoglycans and the very low density lipoprotein receptor. J. Biol. Chem. 276:8934-8941.

77. Jensen, D.R., et al. 1997. Prevention of dietinduced obesity in transgenic mice overexpressing skeletal muscle lipoprotein lipase. Am. J. Physiol. 273:R683-R689.

78. Blanchette-Mackie, E.J., Masuno, H., Dwyer, N.K., Olivecrona, T., and Scow, R.O. 1989. Lipoprotein lipase in myocytes and capillary endothelium of heart: immunocytochemical study. Am. J. Physiol. 256:E818-E828.

79. Hamilton, M.T., Hamilton, D.G., and Zderic, T.W. 2007. Role of low energy expenditure and sitting in obesity, metabolic syndrome, type 2 diabetes, and cardiovascular disease. Diabetes. 56:2655-2667.

80. Levine, J.A., Vander Weg, M.W., Hill, J.O., and Klesges, R.C. 2006. Non-exercise activity thermogenesis: the Crouching Tiger Hidden Dragon of societal weight gain. Arterioscler. Thromb. Vasc. Biol. 26:729-736

81. Drewnowski, A. 2007. The real contribution of added sugars and fats to obesity. Epidemiol. Rev. 29:160-171.

82. Péterfy, M., et al. 2007. Mutations in LMF1 cause combined lipase deficiency and severe hypertriglyceridemia. Nat. Genet. 39:1483-1487.
83. Cisar, L.A., Hoogewerf, A.J., Cupp, M., Rapport, C.A., and Bensadoun, A. 1989. Secretion and degradation of lipoprotein lipase in cultured adipocytes. Binding of lipoprotein lipase to membrane heparan sulfate proteoglycans is necessary for degradation. J. Biol. Chem. 264:1767-1774.

84. Sukonina, V., Lookene, A., Olivecrona, T., and Olivecrona, G. 2006. Angiopoietin-like protein 4 converts lipoprotein lipase to inactive monomers and modulates lipase activity in adipose tissue. Proc. Natl. Acad. Sci. U. S. A. 103:17450-17455.

85. Backhed, F., et al. 2004. The gut microbiota as an environmental factor that regulates fat storage. Proc. Natl. Acad. Sci. U. S. A. 101:15718-15723.

86. Moro, C., Bajpeyi, S., and Smith, S.R. 2008. Determinants of intramyocellular triglyceride turnover: Implications for insulin sensitivity. Am. J. Physiol. 294:E203-E213.

87. Kim, J.K., et al. 2001. Tissue-specific overexpression of lipoprotein lipase causes tissue-specific insulin resistance. Proc. Natl. Acad. Sci. U. S. A 98:7522-7527.

88. Hegarty, B.D., Furler, S.M., Ye, J., Cooney, G.J., and Kraegen, E.W. 2003. The role of intramuscular lipid in insulin resistance. Acta Physiol. Scand. 178:373-383.

89. Lyon, M., Deakin, J.A., and Gallagher, J.T. 1994. Liver heparan sulfate structure. A novel molecular design. J. Biol. Chem. 269:11208-11215

90. Beigneux, A.P., et al. 2007. Glycosylphosphatidylinositol-anchored high-density lipoproteinbinding protein 1 plays a critical role in the lipolytic processing of chylomicrons. Cell Metab. 5:279-291.

91. Hofmann, S.M., et al. 2007. Adipocyte LDL receptor-related protein-1 expression modulates postprandial lipid transport and glucose homeostasis in mice. J. Clin. Invest. 117:3271-3282.

92. Pennacchio, L.A., et al. 2001. An apolipoprotein influencing triglycerides in humans and mice revealed by comparative sequencing. Science. 294:169-173.

93. Marcais, C., et al. 2005. Apoa5 Q139X truncation predisposes to late-onset hyperchylomicronemia due to lipoprotein lipase impairment. J. Clin. Invest. 115:2862-2869.

94. Merkel, M., et al. 2005. Apolipoprotein AV accelerates plasma hydrolysis of triglyceride-rich lipoproteins by interaction with proteoglycan-bound lipoprotein lipase. J. Biol. Chem. 280:21553-21560.

95. Young, S.G., et al. 2007. GPIHBP1: an endothelial cell molecule important for the lipolytic processing of chylomicrons. Curr. Opin. Lipidol. 18:389-396.

96. Ioka, R.X., et al. 2003. Expression cloning and characterization of a novel glycosylphosphatidylinositol-anchored high density lipoprotein-binding protein, GPI-HBP1. J. Biol. Chem. 278:7344-7349.

97. Razani, B., et al. 2002. Caveolin-1-deficient mice are lean, resistant to diet-induced obesity, and show hypertriglyceridemia with adipocyte abnormalities. J. Biol. Chem. 277:8635-8647.

98. Gin, P., et al. 2007. Normal binding of lipoprotein lipase, chylomicrons, and apo-AV to GPIHBP1 containing a G56R amino acid substitution. Biochim. Biophys. Acta. 1771:1464-1468.

99. Fuki, I.V., Meyer, M.E., and Williams, K.J. 2000 Transmembrane and cytoplasmic domains of syndecan mediate a multi-step endocytic pathway involving detergent-insoluble membrane rafts. Biochem. J. 351:607-612

100.Tkachenko, E., Lutgens, E., Stan, R.V., and Simons, M. 2004. Fibroblast growth factor 2 endocytosis in endothelial cells proceed via syndecan-4-dependent activation of Rac1 and a Cdc42-dependent macropinocytic pathway. J. Cell Sci. 117:3189-3199.

101. Mayor, S., Rothberg, K.G., and Maxfield, F.R. 1994. Sequestration of GPI-anchored proteins in caveolae triggered by cross-linking. Science. 264:1948-1951. 102.Sfeir, Z., Ibrahimi, A., Amri, E., Grimaldi, P., and
Abumrad, N. 1997. Regulation of FAT/CD36 gene expression: further evidence in support of a role of the protein in fatty acid binding/transport. Prostaglandins Leukot. Essent. Fatty Acids. 57:17-21.

103.Bonen, A., Tandon, N.N., Glatz, J.F., Luiken, J.J., and Heigenhauser, G.J. 2006. The fatty acid transporter FAT/CD36 is upregulated in subcutaneous and visceral adipose tissues in human obesity and type 2 diabetes. Int. J. Obes. (Lond.) 30:877-883.

104.Pohl, J., Ring, A., Korkmaz, U., Ehehalt, R., and Stremmel, W. 2005. FAT/CD36-mediated longchain fatty acid uptake in adipocytes requires plasma membrane rafts. Mol. Biol. Cell. 16:24-31.

105. Kim, C.A., et al. 2008. Association of a homozygous nonsense caveolin-1 mutation with BerardinelliSeip congenital lipodystrophy. J. Clin. Endocrinol. Metab. 93:1129-1134.

106. Kita, T., et al. 1982. Hepatic uptake of chylomicron remnants in WHHL rabbits: a mechanism genetically distinct from the low density lipoprotein receptor. Proc. Natl. Acad. Sci. U. S. A. 79:3623-3627.

107. Rubinsztein, D.C., et al. 1990. Chylomicron remnant clearance from the plasma is normal in familial hypercholesterolemic homozygotes with defined receptor defects. J. Clin. Invest. 86:1306-1312.

108.Herz, J., et al. 1995. Initial hepatic removal of chylomicron remnants is unaffected but endocytosis is delayed in mice lacking the low density lipoprotein receptor. Proc. Natl. Acad. Sci. U. S. A. 92:4611-4615.

109. Rohlmann, A., Gotthardt, M., Hammer, R.E., and Herz, J. 1998. Inducible inactivation of hepatic LRP gene by cre-mediated recombination confirms role of LRP in clearance of chylomicron remnants. J. Clin. Invest. 101:689-695.

110.van Dijk, M.C.M., et al. 1991. Recognition of chylomicron remnants and beta-migrating very-lowdensity lipoproteins by the remnant receptor of parenchymal liver cells is distinct from the liver alpha 2-macroglobulin-recognition site. Biochem. J. 279:863-870.

111.MacArthur, J.M., et al. 2007. Liver heparan sulfate proteoglycans mediate clearance of triglyceriderich lipoproteins independently of LDL receptor family members. J. Clin. Invest. 117:153-164.

112.Jones, C., et al. 2007. Disruption of LDL but not VLDL clearance in autosomal recessive hypercholesterolemia. J. Clin. Invest. 117:165-174.

113.Ebara, T., et al. 2000. Delayed catabolism of apoB48 lipoproteins due to decreased heparan sulfate proteoglycan production in diabetic mice. J. Clin. Invest. 105:1807-1818.

114.Wilsie, L.C., Gonzales, A.M., and Orlando, R.A. 2006. Syndecan-1 mediates internalization of apoE-VLDL through a low density lipoprotein receptor-related protein (LRP)-independent, nonclathrin-mediated pathway. Lipids Health Dis. 5:23.

115.Fuki, I.V., Iozzo, R.V., and Williams, K.J. 2000. Perlecan heparan sulfate proteoglycan: a novel receptor that mediates a distinct pathway for ligand catabolism. J. Biol. Chem. 275:25742-25750.

116.Argyris, E.G., et al. 2004. The perlecan heparan sulfate proteoglycan mediates cellular uptake of HIV-1 Tat through a pathway responsible for biological activity. Virology. 330:481-486.

117. Belting, M., et al. 2003. Glypican-1 is a vehicle for polyamine uptake in mammalian cells: a pivital role for nitrosothiol-derived nitric oxide. J. Biol. Chem. 278:47181-47189.

118. Roskams, T., et al. 1995. Heparan sulfate proteoglycan expression in normal human liver. Hepatology. 21:950-958.

119.Saarela, J., Rehn, M., Oikarinen, A., Autio-Harmainen, H., and Pihlajaniemi, T. 1998. The short and long forms of type XVIII collagen show clear tissue specificities in their expression and location in basement membrane zones in humans. Am.J. Pathol. 153:611-626. 
120.Comelli, E.M., et al. 2006. A focused microarray approach to functional glycomics: transcriptional regulation of the glycome. Glycobiology. 16:117-131.

121. Dane-Stewart, C.A., et al. 2003. Chylomicron remnant metabolism studied with a new breath test in postmenopausal women with and without type 2 diabetes mellitus. Clin. Endocrinol. (Oxf.) 58:415-420.

122. Williams, K.J., et al. 2005. Loss of heparan $N$-sulfotransferase in diabetic liver: role of angiotensin II. Diabetes. 54:1116-1122.

123.Kjellén, L., Bielefeld, D., and Hook, M. 1983. Reduced sulfation of liver heparan sulfate in exper- imentally diabetic rats. Diabetes. 32:337-342.

124. Olsson, U., et al. 2001. Changes in matrix proteoglycans induced by insulin and fatty acids in hepatic cells may contribute to dyslipidemia of insulin resistance. Diabetes. 50:2126-2132.

125.Duran-Sandoval, D., et al. 2004. Glucose regulates the expression of the farnesoid $\mathrm{X}$ receptor in liver. Diabetes. 53:890-898.

126. Anisfeld, A.M., et al. 2003. Syndecan-1 expression is regulated in an isoform-specific manner by the farnesoid-X receptor. J. Biol. Chem. 278:20420-20428.

127. Martins, I.J., Tran, J.M., and Redgrave, T.G. 2002.
Food restriction normalizes chylomicron remnant metabolism in murine models of obesity as assessed by a novel stable isotope breath test. J. Nutr. 132:176-181.

128. Ybarra, J., James, R.W., Makoundou, V., Bioletto, S., and Golay, A. 2001. Effects of short-term modest weight loss on fasting and post-prandial lipoprotein sub-fractions in type 2 diabetes mellitus patients. Diabetes Metab. 27:701-708.

129.Van Eck, M., et al. 2008. Scavenger receptor BI facilitates the metabolism of very-low-density lipoproteins in vivo. J. Lipid Res. 49:136-146. 